\section{Fragile $X$ testing as a second-tier test}

To the Editor: We read with great interest the article by Weinstein et al. ${ }^{1}$ in which the authors measured the diagnostic yield of chromosomal microarray (CMA) and fragile X (FX) testing in 310 pediatric-aged males with either intellectual disabilities/learning delays (ID/LD) or autism spectrum disorder. Overall, the conclusions of their study are in line with that reported in the literature (15-20\% diagnostic rate for CMA and $\sim 2 \%$ diagnostic rate for FX). The most interesting observation was that all individuals with a positive FX result (full mutations; 2/2) had highly suggestive clinical features and family history. Based on these findings, the authors concluded that while CMA testing continues to be appropriate for pediatric-aged males with ID/LD or autism spectrum disorder, FX testing should be reserved for patients with physical, psychological, and/or a family history suggestive of FX.

In 2016 we recognized the increasing volumes of FX testing performed at our institution but apparent dearth of FX patients identified by or referred to our clinical genetics department. We hypothesized that this observation might be explained by a lower than expected diagnostic yield of FX testing in our patient population. To test this hypothesis, we extracted appropriately de-identified clinical and laboratory information for all individuals referred to the Children's Hospital of Eastern Ontario's Genetics Diagnostic Laboratory for FX testing between years 2010 and 2015, including the reason for referral, age and sex of the patient, and the result (full mutation $(>200$ repeats), premutation (56-200 repeats), or normal $(<56$ repeats)). We conducted a subanalysis of this data focusing, in a similar fashion to Weinstein et al., on 1177 pediatric-aged ( $<19$ years old) males referred for FX testing (Supplementary Figure 1 online). Interestingly, while the number of positive results (full mutation) remained relatively constant between years 2010 and 2015 (between 0 and 4 diagnoses per year), the diagnostic yield decreased over time (from as high as $2.4 \%$ in 2011 to $0 \%$ in 2015); this correlates with gradual increase in the number of samples tested per year (ranging from 124 in 2010 to 255 in 2015). Furthermore, when we consulted the referring providers for the last five positive diagnoses from the Clinical Genetics Department at the Children's Hospital of Eastern Ontario and reviewed the available clinical consultation notes, the diagnosis of FX was strongly suspected based on both clinical presentation and family history in all five instances.

We believe that the increased demand for FX testing is due to more and more patients being "screened" for FX as a first-line test by geneticists, subspecialty pediatricians, and general pediatricians based on recommendations by the American College of Medical Genetics and Genomics and the American
Academy of Pediatrics. ${ }^{2,3}$ While limited by the size of the study population, our data suggests that increased FX screening does not result in increased diagnoses. We therefore support the consideration raised by Weinstein et al. that in the age of CMA, it is time to make FX a second-tier test for patients where the clinical suspicion is low. We would also suggest that in the absence of suggestive features, FX testing should be secondary to next-generation sequencing approaches, including relevant large multigene panel testing and exome sequencing, for the subset of patients with moderate to severe ID, as the diagnostic rate with these approaches will be considerably higher (e.g., $\sim 20 \%$ with exome sequencing). ${ }^{4}$

The current guidelines for FX screening were developed based on evidence at the time that FX was the most common inherited cause of $\mathrm{ID}^{5}$ in the context of limited molecular tests available for other ID-related conditions. Contrary to this, we now recognize over 1,000 genes related to ID (data from www. OMIM.org), making unbiased testing methods with higher likelihoods of a diagnosis, such as CMA and exome sequencing, more appealing. In publicly funded health-care systems with finite resources, requests for genetic testing must be carefully considered; we need to ask whether it is advisable to screen for diseases as first-line in the context of low clinical suspicion when other tests with a much higher yield are available.

\section{SUPPLEMENTARY MATERIAL}

Supplementary material is linked to the online version of the paper at http://www.nature.com/gim

\section{DISCLOSURE}

The authors declare no conflict of interest.

Taila Hartley, MSc, MSc ${ }^{1}$, Ryan Potter, BSc ${ }^{2}$, Lauren Badalato, $M D, M S c^{1,2}$, Amanda C. Smith, $P h D^{1,2}$, Olga Jarinova, $P h D^{1,2}$ and Kym M. Boycott, $M D, P h D^{1,2}$

${ }^{1}$ Children's Hospital of Eastern Ontario Research Institute, University of Ottawa, Ottawa, Ontario, Canada; ${ }^{2}$ Department of Genetics, Children's Hospital of Eastern Ontario, Ottawa, Ontario, Canada. Correspondence: Kym M. Boycott

(kboycott@cheo.on.ca)

\section{REFERENCES}

1. Weinstein $V$, Tanpaiboon $P$, Chapman $K A$, et al. Do the data really support ordering fragile $X$ testing as a first-tier test without clinical features? Genet Med. E-pub ahead of print 25 May, 2017.

2. Shen $Y$, Dies KA, Holm IA, et al. Clinical genetic testing for patients with autism spectrum disorders. Pediatrics 2010; 125:e727-e735.

3. Moeschler JL, Shevell M. Comprehensive evaluation of the child with intellectual disability of global developmental delays. Pediatrics 2014; 134:e903-e918.

4. Hamdam FF, Srour M, Capo-Chichi JM, et al. De novo mutations in moderate or severe intellectual disability. PLoS Genet 2014:10:e1004772.

5. Gallagher A, Hallahn B. Fragile X-associated disorders: a clinical overview. J Neurol 2012;259:401-413.

Advance online publication 14 September 2017. doi:10.1038/gim.2017.147 